\title{
STRONGLY ERGODIC SEQUENCES OF INTEGERS AND THE INDIVIDUAL ERGODIC THEOREM
}

\author{
J. R. BLUM ${ }^{1}$ AND J. I. REICH
}

\begin{abstract}
Let $S=\left\{k_{1}, k_{2}, \ldots\right\}$ be an increasing sequence of positive integers. We call $S$ strongly ergodic if for every measure preserving transformation $T$ on a probability space $(\Omega, \mathcal{F}, P)$ and every $f \in L_{1}(\Omega)$ we have $\lim _{n \rightarrow \infty}(1 / n) \sum_{j=1}^{n} f\left(T^{k_{j}} \omega\right)=P f(\omega)$ a.e. where $P f$ is the appropriate limit guaranteed by the individual ergodic theorem. We give sufficient conditions for a sequence $S$ to be strongly ergodic and provide examples.
\end{abstract}

1. Introduction. Let $S=\left\{k_{1}, k_{2}, \ldots\right\}$ be an increasing sequence of positive integers. We shall call $S$ an ergodic sequence if

$$
\lim _{n \rightarrow \infty} \frac{1}{n} \sum_{j=1}^{n} e^{i k_{j} \alpha}=0 \text { for } 0<\alpha<2 \pi .
$$

To reason for this terminology is as follows: Let $(\Omega, \mathcal{F}, P)$ be a probability space and let $T$ be a bimeasurable, measure preserving transformation mapping $\Omega$ onto $\Omega$. Then if $S$ is an ergodic sequence, we have the mean ergodic theorem holding for $S$, i.e.,

$$
\lim _{n \rightarrow \infty} \frac{1}{n} \sum_{j=1}^{n} f\left(T^{k_{j}} \omega\right)=\operatorname{Pf}(\omega),
$$

for every $f \in L_{2}(\Omega)$, the convergence being $L_{2}$ convergence. Here $P$ is the orthogonal projection onto the subspace $\left\{f \in L_{2}(\Omega) \mid f(T \omega)=f(\omega)\right.$ a.e. $\}$. Conversely, if (1.2) holds for every probability space and every measure preserving transformation, then (1.1) must hold. For details see, e.g., [1].

In this paper we shall be concerned with the individual ergodic theorem. Let us call $S$ strongly ergodic if (1.2) holds almost everywhere for every $f \in L_{1}(\Omega)$. In that case the right-hand side of (1.2) is to be interpreted as the appropriate limit guaranteed by the individual ergodic theorem.

Not every ergodic sequence is strongly ergodic as we shall show by an example. It is easy to see that every strongly ergodic sequence must be ergodic since (1.1) is equivalent to (1.2) restricted to bounded functions. In the next section we construct the example mentioned above. In $\S 3$, we give sufficient conditions for an ergodic sequence to be strongly ergodic and in $\S 4$ we give examples of such sequences. Finally, in the last section we mention some open questions.

Received by the editors February 12, 1982.

1980 Mathematics Subject Classification. Primary 28D05, 47A35, 60F15, 60G10.

Key words and phrases. Individual ergodic theorems, subsequences, strongly ergodic sequences.

${ }^{1}$ Research supported by N.S.F. Grant M.C.S. 800-2179.

(c) 1982 American Mathematical Society 0002-9939/82/0000-0288/\$02.25 
2. An example. The example given here is a minor modification of one given by Emerson [3]. For the sake of completeness we give the details. For each positive integer $n$, let $S_{n}=\sum_{i=1}^{n} 1 /(i+1)(\bmod 1)$. We now construct the sequence $S$ in stages. Let $0<\alpha<1, \alpha$ irrational. In the first stage let $k_{1}=1$. Suppose we have completed the first $n-1$ stages and have defined the integers $k_{1}, \ldots, k_{r}$ which are to be in $S$. Now define $k_{r+1}, \ldots$, by $k_{r+j}=k_{r}+j$ for $j=1, \ldots, n !$. Note that since $\alpha j(\bmod 1)$ is equidistributed we can find arbitrarily large integers $k$ so that $0 \leq\left(S_{n}+k \alpha\right)(\bmod 1) \leq 1 / n$. Choose $\left[n ! / n^{1 / 4}\right]$ such integers all of which are larger than $k_{r+n !}$, where $[x]$ is the largest integer not exceeding $x$, and define them to be $k_{s}$ for $r+n !<s \leq r+n !+\left[n ! / n^{1 / 4}\right]$. This concludes the $n$th stage of defining $S$.

Now let $(\Omega, \mathcal{F}, P)$ be the unit interval with the Borel sets and Lebesgue measure and define $T x=x+\alpha(\bmod 1)$ for $0 \leq x \leq 1$. Then $T$ is known to be measure preserving and ergodic. Let

$$
f(x)= \begin{cases}0, & x=0 \\ 1 / \sqrt{x}, & 0<x \leq 1\end{cases}
$$

Then $f \in L_{1}(\Omega)$. Now choose $x_{0}$ in the unit interval. Then $x_{0}$ lies in infinitely many subintervals $\left[S_{n}, S_{n+1}\right]$. For such an $n$ we have $0 \leq T^{k_{\bullet}} x_{0} \leq 2 / n$ for all $\left[n ! / n^{1 / 4}\right]$ integers $k_{s}$ constructed at the end of the $n$th stage of defining $S$. If $k_{s_{0}}$ is the last such integer we have

$$
\frac{1}{s_{0}} \sum_{j=1}^{s_{0}} f\left(T^{k_{j}} x_{0}\right) \geq \frac{1}{\sum_{j=1}^{n} j !\left(1+1 / j^{1 / 4}\right)}\left(n ! / n^{1 / 4}-1\right) \frac{\sqrt{n}}{\sqrt{2}}
$$

which diverges to $+\infty$. Thus $\lim \sup _{n}\left(1 / k_{n}\right) \sum_{j=1}^{n} f\left(T^{k_{j}} x\right)=+\infty$ for every $x$ in the unit interval.

On the other hand, we show that $S$ is ergodic. To see this, let $S_{n}=\left\{k_{1}, \ldots, k_{n}\right\}$ be the first $n$ elements of $S$ and let $S_{n}+1=\left\{k_{1}+1, \ldots, k_{n}+1\right\}$. Let $|A|$ be the cardinality of $A$. In [1] it is shown that if $\lim _{n \rightarrow \infty}\left|S_{n} \cap S_{n}+1\right| / n=1$ then $S$ is ergodic, and it is easy to verify that our sequence $S$ has this property.

3. Strongly ergodic sequences. Let $S=\left\{k_{1}, k_{2}, \ldots\right\}$ be a sequence. Let $J$ be a closed subinterval of the open interval $(0,2 \pi)$. Following Reich [5] we shall say that $S$ satisfies a uniform order condition on $J$ if there exists an infinite sequence $\left\{N_{r}\right\}$ of positive integers such that

$$
\begin{aligned}
& \text { (a) } \sum_{r=1}^{\infty} \sup _{\alpha \in J}\left|\frac{1}{N_{r}} \sum_{j=1}^{N_{r}} e^{i k_{j} \alpha}\right|^{2}<\infty \text { and } \\
& \text { (b) } \lim _{r \rightarrow \infty} \frac{N_{r}}{N_{r+1}}=1 .
\end{aligned}
$$

Let $J$ be the class of intervals $J$ for which $S$ satisfies a uniform order condition. Let $K=[0,2 \pi] \cap\left(\bigcup_{J \in J}(\text { interior } J)\right)^{c}$. We shall say that $S$ saturates if $\mathcal{K}$ is at most countable. Note that $\mathcal{K}$ always contains $\{0,2 \pi\}$. For example if $S=\{1,2, \ldots\}$ then $K=\{0,2 \pi\}$. If $S$ consists of the even integers then $K=\{0, \pi, 2 \pi\}$, etc.

Concerning saturating sequences Reich [5] has proved the following result. Let $(\Omega, \mathcal{F}, P)$ and $T$ be as in $\S 1$, and suppose $T$ is mixing. Then there exists a dense 
subspace $D_{T}$ of $L_{2}(\Omega)$ such that for $f \in D_{T}$ we have

$$
\lim _{n \rightarrow \infty} \frac{1}{n} \sum_{j=1}^{n} f\left(T^{k_{j}} \omega\right)=P f(\omega) \text { a.e. }
$$

Now suppose $T$ is measure preserving and 1-1 onto. Let $U$ be the associated unitary operator defined on $L_{2}(\Omega)$ by $U f(\omega)=f(T \omega)$ for $f \in L_{2}(\Omega)$. Let $L$ be the subspace of $L_{2}(\Omega)$ defined by $L=\left\{\sum_{i=0}^{n} f_{i}\right\}$ where the spectral measure of $f_{0}$ is continuous and $f_{1}, \ldots, f_{n}$ are eigenfunctions of $U$ corresponding to eigenvalues, say $e^{i \lambda_{1}}, \ldots, e^{i \lambda_{n}}$ of $U$. The following lemma is elementary and will not be proved here.

LEMMA. $L$ is dense in $L_{2}(\Omega)$.

Using the lemma we can now prove

THEOREM 1. Let $S$ be an ergodic sequence and suppose $S$ saturates. Let $T$ be a 1-1, bimeasurable and measure preserving transformation of $\Omega$ onto $\Omega$. Then there exists a dense subspace of $L_{2}(\Omega)$ such that (3.2) holds for each $f$ in the subspace.

Proof. We shall prove the theorem for $f \in L$. Assume w.l.o.g. that $\int_{\Omega} f d P=$ 0 . If $f$ has continuous spectral measure, then the proof by Reich [5, Theorem (4.1)] applies. If $f$ is an eigenfunction with eigenvalue $e^{i \lambda}, 0<\lambda<2 \pi$, then $(1 / n) \sum_{j=1}^{n} f\left(T^{k_{j}} \omega\right)=f(\omega)(1 / n) \sum_{j=1}^{n} e^{i k_{j} \lambda} \rightarrow 0$ for every $\omega \in \Omega$, since $S$ is ergodic. The theorem then follows from the structure of $L$.

In order to extend Theorem 1 to $L_{1}(\Omega)$, we shall need the following special case of a theorem which may be found in Garsia [4]. have

THEOREM. Suppose there exists a constant $C>0$ such that for all $f \in L_{1}(\Omega)$ we

$$
P\left\{\omega\left|\sup _{n \geq 1}\right| \frac{1}{n} \sum f\left(T^{k_{j}} \omega\right) \mid>\lambda\right\} \leq \frac{C\|f\|_{1}}{\lambda}
$$

Then the set $\left\{f \in L_{1}(\Omega) \mid \lim _{n \rightarrow \infty}(1 / n) \sum_{j=1}^{n} f\left(T^{k_{j}} \omega\right)\right.$ exists a.e. $\}$ is closed in $L_{1}(\Omega)$.

Now let $S$ be a sequence and let $\chi_{S}(\cdot)$ be the indicator function of $S$. We shall say that $S$ has positive lower density if $\liminf _{n \rightarrow \infty}(1 / n) \sum_{j=1}^{n} \chi_{S}(j)>0$. We can now state our main result.

THEOREM 2. Let $S$ be a sequence and suppose $S$ satisfies

$$
\begin{aligned}
& \text { (i) } S \text { is ergodic, } \\
& \text { (ii) } S \text { has positive lower density, } \\
& \text { (iii) } S \text { saturates. }
\end{aligned}
$$

Let $T$ be a 1-1, bimeasurable and measure preserving transformation of $\Omega$ onto $\Omega$. Then $\lim _{n \rightarrow \infty}(1 / n) \sum_{j=1}^{n} f\left(T^{k_{j}} \omega\right)=P f(\omega)$ a.e. for every $f \in L_{1}(\Omega)$.

Here $P f$ is to be interpreted as the appropriate limit guaranteed by the individual ergodic theorem.

ProOF. Suppose $S=\left\{k_{1}, k_{2}, \ldots\right\}$ satisfies (3.4). Let $M=\sup _{n \geq 1}\left(k_{n} / n\right)<\infty$. Then for $f \in L_{1}(\Omega)$ we have

$$
P\left\{\omega\left|\sup _{n \geq 1} \frac{1}{n}\right| \sum_{j=1}^{n} f\left(T^{k_{j}} \omega\right) \mid>\lambda\right\} \leq P\left\{\omega\left|\sup _{n \geq 1} \frac{1}{k_{n}} \sum_{j=1}^{k_{n}}\right| f\left(T^{j} \omega\right) \mid>\frac{\lambda}{\mu}\right\} \leq \frac{M\|f\|_{1}}{\lambda}
$$

by the maximal ergodic lemma. 
Hence (3.3) applies and the set of a.e. convergence is closed in $L_{1}(\Omega)$. On the other hand by Theorem 1, this set includes $L$ which is dense in $L_{2}(\Omega)$ and hence in $L_{1}(\Omega)$. It is easy to verify that the appropriate limit is obtained.

In order to apply Theorem 2 we shall need a useful criterion to verify that a sequence saturates. Such a criterion is given by Reich [5]. We have

THEOREM. Let $S=\left\{k_{1}, k_{2}, \ldots\right\}$ be a sequence. Suppose there exists a positive integer $r$ and $a$ number $\alpha>0$ such that

$$
\left(\frac{\left|\left\{k_{1}, \ldots, k_{n}\right\} \cap\left\{k_{1}+r, \ldots, k_{n}+r\right\}\right|}{n}-1\right)=O\left(\frac{1}{n^{\alpha}}\right) .
$$

Then $S$ saturates.

4. Examples. Here is a simple example to which Theorem 2 applies. Let $S^{\prime}=$ $\{2,4, \ldots\}$ be the set of even positive integers. Then $S^{\prime}$ has positive lower density and saturates. However, $S^{\prime}$ is not ergodic. We now modify $S^{\prime}$ as follows. When $n$ is even we keep the elements of $S^{\prime}$ between $n^{2}$ and $(n+1)^{2}$ when $n$ is odd we add one to each element of $S^{1}$ between $n^{2}$ and $(n+1)^{2}$. Let $S$ be the resulting sequence. Then it can be shown by the last theorem that Theorem 2 applies. Similarly we can modify any sequence $S^{\prime}=\{k, 2 k, 3 k, \ldots\}$ where $k$ is a positive integer.

A richer class of examples can be found by constructing random sequences. Let $\left\{Y_{n}, n=1,2, \ldots\right\}$ be a sequence of independent, identically distributed random variables which are positive with probability one and integer-valued. Assume that $Y_{1}$ is nonlattice so that $E\left(e^{i \alpha Y_{1}}\right) \neq 1$ for $0<\alpha<2 \pi$. Let $S_{n}=\sum_{j=1}^{n} Y_{j}$. The following theorem was proved by Blum and Cogburn [2].

THEOREM. Suppose EY $Y_{1}<\infty$ and let $J$ be a closed subinterval of $(0,2 \pi)$. Then there exists a set $C$ of sample sequences $\left\{Y_{n}, n=1,2, \ldots\right\}$ with $P\{C\}=1$ such that if $\left\{Y_{n}, n=1,2, \ldots\right\} \in C$ we have

$$
\sup _{\alpha \in J}\left|\frac{1}{n} \sum_{j=1}^{n} e^{S_{j} \alpha}\right|=O\left(\frac{1}{n^{1 / 8}}\right) .
$$

From this we can deduce

THEOREM 3. If $E Y_{1}<\infty$ and $Y_{1}$ is nonlattice, then for almost all sequences $\left\{Y_{n}, n=1,2, \ldots\right\}$ the associated sequence of partial sums $\left\{S_{n}\right\}$ saturates.

PROOF. Let $J_{n}=[1 / n, 2 \pi-1 / n]$. Then by the above theorem there exists a set of sequences $C_{n}$ with $P\left\{C_{n}\right\}=1, n=1,2, \ldots$, such that the sequences $\left\{S_{k}\right\}$ corresponding to sequences in $C_{n}$ satisfy a uniform order condition on $J_{n}$. Let $C=\bigcap_{n=1}^{\infty} C_{n}$. Then $P\{C\}=1$ and it is clear that for $\left\{Y_{n}\right\} \in C$, the associated $\left\{S_{k}\right\}$ sequence saturates.

Finally we have

THEOREM 4. Suppose $E Y_{1}<\infty$ and $Y_{1}$ is nonlattice. Then there exists a set of sample sequences $\left\{Y_{n}, n=1,2, \ldots\right\}$ of probability one such that if $\left\{S_{n}\right\}$ is the corresponding sequence of partial sums and $T$ is any 1-1, bimeasurable and measure preserving transformation of $\Omega$ onto $\Omega$ we have

$$
\lim _{n \rightarrow \infty} \frac{1}{n} \sum_{j=1}^{n} f\left(T^{S_{j}} \omega\right)=\operatorname{Pf}(\omega) \text { a.e. }
$$

for every $f \in L_{1}(\Omega)$. 
Proof. Let $C$ be the set of sample sequences of Theorem 3. Let $C^{\prime}$ be the set of sample sequences for which $\lim _{n \rightarrow \infty}(1 / n) \sum_{j=1}^{n} Y_{j}=E Y_{1}$. Then $D=C \cap C^{\prime}$ has probability one, and it is clear that every sequence $\left\{S_{k}\right\}$ corresponding to a sample sequence $\left\{Y_{n}\right\} \in D$ satisfies the hypotheses of Theorem 2 .

5. Concluding remarks. As mentioned earlier, every strongly ergodic sequence $S$ must be ergodic. However, we do not know of any other necessary conditions for strong ergodicity. In particular, our method fails when $S$ has density zero. Here is a simple example of a sequence which is ergodic and saturates. Let $S=$ $\{1, \ldots, n !, n !+1, n !+2, \ldots, n !+n,(n+1) !,(n+1) !+1, \ldots\}$. Clearly $S$ has density zero. We have been unable to decide whether $S$ is strongly ergodic.

Dedication. This paper is dedicated to the memory of Julius Reuven Blum who died in the spring of 82 . He was a wonderful friend, an outstanding mathematician and a warm persion who cared for others. He leaves a void that will be difficult to fill.

\section{REFERENCES}

1. J. R. Blum and B. Eisenberg, Generalized summing sequences and the mean ergodic theorem, Proc. Amer. Math. Soc. 42 (1974), 423-429.

2. J. R. Blum and R. Cogburn, On ergodic sequences of measures, Proc. Amer. Math. Soc. 51 (1975), 359-365.

3. W. R. Emerson, The pointwise ergodic theorem for amenable groups, Amer. J. Math. 96 (1974), $472-487$.

4. A. M. Garsia, Topics in almost everywhere convergence, Markham, Chicago, Ill., 1970.

5. J. I. Reich, On the individual ergodic theorem for subsequences, Ann. Probability 5 (1977), 10391046.

Division of Statistics, University of California, Davis, California 95616

Department of Mathematics, Baruch College, New York, NeW York 10010 